\title{
Statistical convergence in a paranormed space
}

\author{
Abdullah Alotaibi and Abdullah M Alroqi
}

* Correspondence: aalotaibi@kau.
edu.sa
Department of Mathematics, King
Abdulaziz University, P.O. Box
80203, Jeddah 21589, Saudi Arabia

\begin{abstract}
In this article, we define the notion of statistical convergence, statistical Cauchy and strongly $p$-Cesàro summability in a paranormed space. We establish some relations between them.

AMS subject classification (2000): 41A10; 41A25; 41A36; 40A05; 40A30.
\end{abstract}

Keywords: density, statistical convergence, statistical Cauchy, para-normed space, strongly $p$-Cesàro summability.

\section{Introduction and preliminaries}

The concept of statistical convergence for sequences of real numbers was introduced by Fast [1] and Steinhaus [2] independently in the same year 1951 and since then several generalizations and applications of this notion have been investigated by various authors, namely [3-11]. This notion was defined in normed spaces by Kolk [12] and in locally convex Hausdorff topological spaces by Maddox [13]. Çakalli [14] extended this notation to topological Hausdorff groups. Recently, in [15,16], the concept of statistical convergence is studied in probabilistic normed space and in intuitionistic fuzzy normed spaces, respectively. In this article, we shall study the concept of statistical convergence, statistical Cauchy, and strongly $p$-Cesàro summability in a paranormed space.

Let $K$ be a subset of the set of natural numbers $\mathbb{N}$. Then the asymptotic density of $K$ denoted by $\delta(K)$, is defined as $\delta(K)=\lim _{n} \frac{1}{n}|\{k \leq n: k \in K\}|$, where the vertical bars denote the cardinality of the enclosed set.

A number sequence $x=\left(x_{k}\right)$ is said to be statistically convergent to the number $L$ if for each $\epsilon>0$, the set $K(\epsilon)=\left\{k \leq n:\left|x_{k}-L\right|>\epsilon\right\}$ has asymptotic density zero, i.e.,

$$
\lim _{n} \frac{1}{n}\left|\left\{k \leq n:\left|x_{k}-L\right|\right\}\right|=0 .
$$

In this case we write $s t-\lim x=L$.

A number sequence $x=\left(x_{k}\right)$ is said to be statistically Cauchy sequence if for every $\epsilon>0$, there exists a number $N=N(\epsilon)$ such that

$$
\lim _{n} \frac{1}{n}\left|\left\{j \leq n:\left|x_{j}-x_{N}\right| \geq \varepsilon\right\}\right|=0
$$

The concept of paranorm is a generalization of absolute value (see [17]).

\section{Springer}

(C) 2012 Alotaibi and Altheabi et al; licensee Springer. This is an open access article distributed under the terms of the Creative Commons Attribution License (http://creativecommons.org/licenses/by/2.0), which permits unrestricted use, distribution, and reproduction in any medium, provided the original work is properly cited. 
A paranorm is a function $g: X \rightarrow \mathbb{R}$ defined on a linear space $X$ such that for all $x, y$, $z \in X$

(P1) $g(x)=0$ if $x=\theta$

(P2) $g(-x)=g(x)$

(P3) $g(x+y) \leq g(x)+g(y)$

(P4) If $\left(\alpha_{n}\right)$ is a sequence of scalars with $\alpha_{n} \rightarrow \alpha_{0}(n \rightarrow \infty)$ and $x_{n}, a \in X$ with $x_{n} \rightarrow a(n \rightarrow \infty)$ in the sense that $g\left(x_{n}-a\right) \rightarrow 0(n \rightarrow \infty)$, then $\alpha_{n} x_{n} \rightarrow \alpha_{0} a$ $(n \rightarrow \infty)$, in the sense that $g\left(\alpha_{n} x_{n}-\alpha_{0} a\right) \rightarrow 0(n \rightarrow \infty)$.

A paranorm $g$ for which $g(x)=0$ implies $x=\theta$ is called a total paranorm on $X$, and the pair $(X, g)$ is called a total paranormed space.

Note that each seminorm (norm) $p$ on $X$ is a paranorm (total) but converse need not be true.

In this article, we define and study the notion of convergence, statistical convergence, statistical Cauchy, and strong summability by a modulus function in a paranormed space.

Let $(X, g)$ be a paranormed space.

Definition 1.1. A sequence $x=\left(x_{k}\right)$ is said to be convergent (or $g$-convergent) to the number $\xi$ in $(X, g)$ if for every $\varepsilon>0$, there exists a positive integer $k_{0}$ such that $g\left(x_{k}-\xi\right)$ $<\varepsilon$ whenever $k \geq k_{0}$. In this we write $g-\lim x=\xi$, and $\xi$ is called the $g$-limit of $x$.

Definition 1.2. A sequence $x=\left(x_{k}\right)$ is said to be statistically convergent to the number $\xi$ in $(X, g)$ (or $g($ st)-convergent) if for each $\epsilon>0$,

$$
\lim _{n} \frac{1}{n}\left|\left\{k \leq n: g\left(x_{k}-\xi\right)>\varepsilon\right\}\right|=0 .
$$

In this case, we write $g(s t)-\lim x=\xi$. We denote the set of all $g(s t)$-convergent sequences by $S_{g}$.

Definition 1.3. A number sequence $x=\left(x_{k}\right)$ is said to be statistically Cauchy sequence in $(X, g)$ (or $g(s t)$-Cauchy) if for every $\epsilon>0$ there exists a number $N=N(\epsilon)$ such that

$$
\lim _{n} \frac{1}{n}\left|\left\{j \leq n: g\left(x_{j}-x_{N}\right) \geq \varepsilon\right\}\right|=0 .
$$

\section{Main results}

Theorem 2.1. If a sequence $x=\left(x_{k}\right)$ is statistically convergent in $(X, g)$ then $g(s t)$-limit is unique.

Proof. Suppose that $g(s t)-\lim x=\xi_{1}$ and $g(s t)-\lim x=\xi_{2}$. Given $\varepsilon>0$, define the following sets as:

$$
\begin{aligned}
& K_{1}(\varepsilon)=\left\{n \in \mathbb{N}: g\left(x_{n}-\xi_{1}\right) \geq \varepsilon / 2\right\}, \\
& K_{2}(\varepsilon)=\left\{n \in \mathbb{N}: g\left(x_{n}-\xi_{2}\right) \geq \varepsilon / 2\right\} .
\end{aligned}
$$

Since $g(s t)-\lim x=\xi_{1}$, we have $\delta\left(K_{1}(\varepsilon)\right)=0$. Similarly, $g(s t)$ - $\lim x=\xi_{2}$ implies that $\delta\left(K_{2}(\varepsilon)\right)=0$. Now, let $K(\varepsilon)=K_{1}(\varepsilon) \cup K_{2}(\varepsilon)$. Then $\delta(K(\varepsilon))=0$ and hence the compliment $K^{C}(\varepsilon)$ is a nonempty set and $\delta\left(K^{C}(\varepsilon)\right)=1$. Now if $k \in \mathbb{N} \backslash K(\varepsilon)$, then we have $g\left(\xi_{1}-\xi_{2}\right) \leq g$ $\left(x_{n}-\xi_{1}\right)+g\left(x_{n}-\xi_{2}\right)<\varepsilon / 2+\varepsilon / 2=\varepsilon$. 
Since $\varepsilon>0$ was arbitrary, we get $g\left(\xi_{1}-\xi_{2}\right)=0$ and hence $\xi_{1}=\xi_{2}$.

Theorem 2.2. If $g$ - $\lim x=\xi$ then $g(s t)-\lim x=\xi$ but converse need not be true in general.

Proof. Let $g$ - $\lim x=\xi$. Then for every $\varepsilon>0$, there is a positive integer $N$ such that

$$
g\left(x_{n}-\xi\right)<\varepsilon
$$

for all $n \geq N$. Since the set $A(\epsilon):=\left\{k \in \mathbb{N}: g\left(x_{k}-\xi\right) \geq \varepsilon\right\} \subset\{1,2,3, \ldots\}, \delta(A(\epsilon))=0$. Hence $g(s t)-\lim x=\xi$.

The following examle shows that the converse need not be true.

Example 3.1. Let $X=\ell(1 / k):=\left\{x=\left(x_{k}\right): \sum_{k}\left|x_{k}\right|^{1 / k}<\infty\right\}$ with the paranorm $g(x)=\left(\sum_{k}\right.$ $\left.\left|x_{k}\right|^{1 / k}\right)$. Define a sequence $x=\left(x_{k}\right)$ by

$$
x_{k}:=\left\{\begin{array}{l}
k, \text { if } k=n^{2}, n \in \mathbb{N} ; \\
0, \quad \text { otherwise }
\end{array}\right.
$$

and write

$$
K(\varepsilon):=\left\{k \leq n: g\left(x_{k}\right) \geq \varepsilon\right\}, 0<\varepsilon<1 .
$$

We see that

$$
g\left(x_{k}\right):= \begin{cases}k^{1 / k}, & \text { if } k=n^{2}, n \in \mathbb{N} ; \\ 0, & \text { otherwise }\end{cases}
$$

and hence

$$
\lim _{k} g\left(x_{k}\right):=\left\{\begin{array}{l}
1, \text { if } k=n^{2}, n \in \mathbb{N} ; \\
0, \text { otherwise }
\end{array}\right.
$$

Therefore $g-\lim x$ does not exist. On the other hand $\delta(K(\varepsilon))=0$, that is, $g(s t)-\lim x=$ 0 .

Theorem 2.3. Let $g(s t)-\lim x=\xi_{1}$ and $g(s t)-\lim y=\xi_{2}$. Then

(i) $g(s t)-\lim (x \pm y)=\xi_{1} \pm \xi_{2}$,

(ii) $g(s t)-\lim \alpha x=\alpha \xi_{1}, \alpha \in \mathbb{R}$.

Proof. It is easy to prove.

Theorem 2.4. A sequence $x=\left(x_{k}\right)$ in $(X, g)$ is statistically convergent to $\xi$ if and only if there exists a set $K=\left\{k_{1}<k_{2}<\ldots<k_{n}<\ldots\right\} \subseteq \mathbb{N}$ with $\delta(K)=1$ such that $g\left(x_{k_{n}}-\xi\right) \rightarrow 0(n \rightarrow \infty)$.

Proof. Suppose that $g(s t)-\lim x=\xi$. Now, write for $r=1,2, \ldots$.

$$
K_{r}:=\left\{n \in \mathbb{N}: g\left(x_{k_{n}}-\xi\right) \leq 1-\frac{1}{r}\right\},
$$

and

$$
M_{r}:=\left\{n \in \mathbb{N}: g\left(x_{k_{n}}-\xi\right)>\frac{1}{r}\right\} .
$$

Then $\delta\left(K_{r}\right)=0$,

$$
M_{1} \supset M_{2} \supset \ldots \supset M_{i} \supset M_{i+1} \supset \ldots,
$$


and

$$
\delta\left(M_{r}\right)=1, r=1,2, \ldots
$$

Now we have to show that for $n \in M_{r},\left(x_{k_{n}}\right)$ is $g$-convergent to $\xi$. On contrary suppose that $\left(x_{k_{n}}\right)$ is not $g$-convergent to $\xi$. Therefore there is $\varepsilon>0$ such that $g\left(x_{k_{n}}-\xi\right) \leq \varepsilon$ for infinitely many terms. Let $M_{\varepsilon}:=\left\{n \in \mathbb{N}: g\left(x_{k_{n}}-\xi\right)>\varepsilon\right\}$ and $\varepsilon>\frac{1}{r}, r \in \mathbb{N}$. Then

$$
\delta\left(M_{\varepsilon}\right)=0,
$$

and by (2.4.1), $M_{r} \subset M_{\varepsilon}$. Hence $\delta\left(M_{r}\right)=0$, which contradicts (2.4.2) and we get that $\left(x_{k_{n}}\right)$ is $g$-convergent to $\xi$.

Conversely, suppose that there exists a set $K=\left\{k_{1}<k_{2}<k_{3}<\ldots<k_{n}<\ldots\right\}$ with $\delta(K)=$ 1 such that $g-\lim _{n \rightarrow \infty} x_{k_{n}}=\xi$. Then there is a positive integer $N$ such that $g\left(x_{n}-\xi\right)<\varepsilon$ for $n>N$. Put $K_{\varepsilon}(t):=\left\{n \in \mathbb{N}: g\left(x_{n}-\xi\right) \geq \varepsilon\right\}$ and $K^{\prime}:=\left\{k_{N+1}, k_{N+2}, \ldots\right\}$. Then $\delta\left(K^{\prime}\right)=1$ and $K_{\varepsilon} \subseteq \mathbb{N}$ - $K^{\prime}$ which implies that $\delta\left(K_{\varepsilon}\right)=0$. Hence $g(s t)$ - $\lim x=\xi$.

Theorem 2.5. Let $(X, g)$ be a complete paranormed space. Then a sequence $x=\left(x_{k}\right)$ of points in $(X, g)$ is statistically convergent if and only if it is statistically Cauchy.

Proof. Suppose that $g(s t)-\lim x=\xi$. Then, we get

$$
\delta(A(\varepsilon))=0,
$$

where $A(\varepsilon):=\left\{n \in \mathbb{N}: g\left(x_{n}-\xi\right) \geq \varepsilon / 2\right\}$. This implies that

$$
\delta\left(A^{C}(\varepsilon)\right)=\delta\left(\left\{n \in \mathbb{N}: g\left(x_{n}-\xi\right)<\varepsilon\right\}\right)=1 .
$$

Let $m \in A^{C}(\varepsilon)$. Then $g\left(x_{m}-\xi\right)<\varepsilon / 2$. Now, let $B(\varepsilon):=\left\{n \in \mathbb{N}: g\left(x_{m}-x_{n}\right) \geq \varepsilon\right\}$. We need to show that $B(\varepsilon) \subset A(\varepsilon)$. Let $n \in B(\varepsilon)$. Then $g\left(x_{n}-x_{m}\right) \geq \varepsilon$ and hence $g\left(x_{n}-\xi\right) \geq$ $\varepsilon / 2$, i.e. $n \in A(\varepsilon)$. Otherwise, if $g\left(x_{n}-\xi\right)<\varepsilon$ then

$$
\varepsilon \leq g\left(x_{n}-x_{m}\right) \leq g\left(x_{n}-\xi\right)+g\left(x_{m}-\xi\right)<\frac{\varepsilon}{2}+\frac{\varepsilon}{2}=\varepsilon,
$$

which is not possible. Hence $B(\varepsilon) \subset A(\varepsilon)$, which implies that $x=\left(x_{k}\right)$ is $g(s t)$ convergent.

Conversely, suppose that $x=\left(x_{k}\right)$ is $g(s t)$-Cauchy but not $g(s t)$-convergent. Then there exists $M \in \mathbb{N}$ such that $\delta(G(\varepsilon)=0$,

where $G(\varepsilon):=\left\{n \in \mathbb{N}: g\left(x_{n}-x_{M}\right) \geq \varepsilon\right\}$, and $\delta(D(\varepsilon))=0$, where $D(\varepsilon):=\left\{n \in \mathbb{N}: g\left(x_{n}-\xi\right)\right.$ $<\varepsilon / 2\}$, i.e., $\delta\left(D^{C}(\varepsilon)\right)=1$. Since $g\left(x_{n}-x_{m}\right) \leq 2 g\left(x_{n}-\xi\right)<\varepsilon$,

if $g\left(x_{n}-\xi\right)<\varepsilon / 2$. Therefore $\delta\left(G^{C}(\varepsilon)\right)=0$, i.e., $\delta(G(\varepsilon)=1$, which leads to a contradiction, since $x=\left(x_{k}\right)$ was $g(s t)$-Cauchy. Hence $x=\left(x_{k}\right)$ must be $g(s t)$-convergent.

\section{Strong summability}

In this section, we define the notion of strong summability by a modulus function and establish its relation with statistical convergence in a paranormed space.

Definition 3.1. A sequence $x=\left(x_{k}\right)$ is said to be strongly $p$-Cesàro summable $(0<p<\infty)$ to the limit $\xi$ in $(X, g)$ if

$$
\lim _{n} \frac{1}{n} \sum_{j=1}^{n}\left(g\left(x_{j}-\xi\right)\right)^{p}=0
$$

and we write it as $x_{k} \rightarrow \xi\left[C_{1}, g\right]_{p}$. In this case $\xi$ is called the $\left[C_{1}, g\right]_{p}$-limit of $x$. 
Theorem 3.1. (a) If $0<p<\infty$ and $x_{k} \rightarrow \xi\left[C_{1}, g\right]_{p}$, then $x=\left(x_{k}\right)$ is statistically convergent to $\xi$ in $(X, g)$.

(b) If $x=\left(x_{k}\right)$ is bounded and statistically convergent to $\xi$ in $(X, g)$ then $x_{k} \rightarrow \xi\left[C_{1}\right.$, $g]_{p}$.

Proof. (a) Let $x_{k} \rightarrow \xi\left[C_{1}, g\right]_{p}$, then

$$
\begin{aligned}
0 \leftarrow \frac{1}{n} \sum_{k=1}^{n}\left(g\left(x_{k}-\xi\right)\right)^{p} \geq & \frac{1}{n} \sum_{\substack{k=1 \\
\left(g\left(x_{k}-\xi\right)\right)^{p} \geq \varepsilon}}^{n}\left(g\left(x_{k}-\xi\right)\right)^{p} \\
\geq & \frac{\varepsilon^{p}}{n}\left|K_{\varepsilon}\right|,
\end{aligned}
$$

as $n \rightarrow \infty$. That is, $\lim _{n \rightarrow \infty} \frac{1}{n}\left|K_{\varepsilon}\right|=0$ and so $\delta\left(K_{\varepsilon}\right)=0$, where $K_{\varepsilon}:=\left\{k \leq n:\left(g\left(x_{k}-\xi\right)\right)^{p}\right.$ $\geq \varepsilon\}$. Hence $x=\left(x_{k}\right)$ is statistically convergent to $\xi$ in $(X, g)$.

(b) Suppose that $x=\left(x_{k}\right)$ is bounded and statistically convergent to $\xi$ in $(X, g)$. Then for $\varepsilon>0$, we have $\delta\left(K_{\varepsilon}\right)=0$. Since $x \in l_{\infty}$, there exists $M>0$ such that $g\left(x_{k}-\xi\right) \leq M$ $(k=1,2, \ldots)$. We have

$$
\frac{1}{n} \sum_{k=1}^{n}\left(g\left(x_{k}-\xi\right)\right)^{p}=\frac{1}{n} \sum_{\substack{k=1 \\ k \notin K_{\varepsilon}}}^{n}\left(g\left(x_{k}-\xi\right)\right)^{p}+\frac{1}{n} \sum_{\substack{k=1 \\ k \in K_{\varepsilon}}}^{n}\left(g\left(x_{k}-\xi\right)\right)^{p}=S_{1}(n)+S_{2}(n),
$$

where

$$
S_{1}(n)=\frac{1}{n} \sum_{\substack{k=1 \\ k \notin K_{\varepsilon}}}^{n}\left(g\left(x_{k}-\xi\right)\right)^{p} \text { and } S_{2}(n)=\frac{1}{n} \sum_{\substack{k=1 \\ k \in K_{\varepsilon}}}^{n}\left(g\left(x_{k}-\xi\right)\right)^{p} .
$$

Now if $k \notin K_{\varepsilon}$ then $S_{1}(n)<\varepsilon^{q}$. For $k \in K_{\varepsilon}$, we have

$$
S_{2}(n) \leq\left(\sup g\left(x_{k}-\xi\right)\right)\left(\left|K_{\varepsilon}\right| / n\right) \leq M\left|K_{\varepsilon}\right| / n \rightarrow 0,
$$

as $n \rightarrow \infty$, since $\delta\left(K_{\varepsilon}\right)=0$. Hence $x_{k} \rightarrow \xi\left[C_{1}, g\right]_{p}$.

This completes the proof of the theorem.

Recall that a modulus $f$ is a function from $[0, \infty)$ to $[0, \infty)$ such that $(i) f(x)=0$ if and only if $x=0$, (ii) $f(x+y) \leq f(x)+f(y)$ for all $x, y \geq 0$, (iii) $f$ is increasing, and (iv) $f$ is continuous from the right at 0 .

Now we define the following:

Definition 3.2. Let $f$ be a modulus. we say that a sequence $x=\left(x_{k}\right)$ is strongly $p$-Cesàro summable with respect to $f$ to the limit $\xi$ in $(X, g)$ if

$$
\lim _{n} \frac{1}{n} \sum_{j=1}^{n} f\left(\left(g\left(x_{j}-\xi\right)\right)^{p}\right)=0
$$

$(0<p<\infty)$. In this case we write $x_{k} \rightarrow \xi(w(f, g, p))$.

As in [13], it is easy to prove the following:

Theorem 3.2. (a) Let $f$ be any modulus and $x_{k} \rightarrow \xi(w(f, g, p))$. Then $x=\left(x_{k}\right)$ is statistically convergent to $\xi$ in $(X, g)$.

(b) $S_{g}=w(f, g, p)$ If and only if $f$ is bounded. 


\section{Acknowledgements}

The authors would like to thank the Deanship of Scientific Research at King Abdulaziz University for its financial support under a grant with number 156/130/1431.

\section{Authors' contributions}

The authors have equitably contributed in obtaining the new results presented in this article. All authors read and approved the final manuscript.

\section{Competing interests}

The authors declare that they have no competing interests.

Received: 14 August 2011 Accepted: 21 February 2012 Published: 21 February 2012

\section{References}

1. Fast, H: Sur la convergence statistique. Colloq Math. 2, 241-244 (1951)

2. Steinhaus, H: Sur la convergence ordinaire et la convergence asymptotique. Collog Math. 2, 73-34 (1951)

3. Osama, H, Edely, H, Mursaleen, M: On statistical A-summability. Math Comput Model. 49, 672-680 (2009). doi:10.1016/j. mcm.2008.05.053

4. Fridy, JA: On statistical convergence. Analysis. 5, 301-313 (1985)

5. Fridy, JA: Lacunary statistical summability. J Math Anal Appl. 173, 497-504 (1993). doi:10.1006/jmaa.1993.1082

6. Moricz, F: Statistical convergence of multiple sequences. Arch Math. 81, 82-89 (2003). doi:10.1007/s00013-003-0506-9

7. Mursaleen, M: $\lambda$-statistical convergence. Math Slovaca. 50, 111-115 (2000)

8. Mursaleen, M, Osama, H, Edely, H: Statistical convergence of double sequences. J Math Anal Appl. 288, 223-231 (2003). doi:10.1016/j.jmaa.2003.08.004

9. Mursaleen, M, Mohiuddine, SA: Statistical convergence of double sequences in intuitionistic fuzzy normed spaces. Chaos Solitons Fractals. 41, 2414-2421 (2009). doi:10.1016/j.chaos.2008.09.018

10. Mursaleen, M, Mohiuddine, SA: On lacunary statistical convergence with respect to the intuitionistic fuzzy normed space. J Comput Appl Math. 233, 142-149 (2009). doi:10.1016/j.cam.2009.07.005

11. Šalát, T: On statistically convergent sequences of real numbers. Math Slovaca. 30, 139-150 (1980)

12. Kolk, E: The statistical convergence in Banach spaces. Tartu UI Toime. 928, 41-52 (1991)

13. Maddox, IJ: Statistical convergence in a locally convex space. Math Cambridge Phil Soc. 104, 141-145 (1988). doi:10.1017/S0305004100065312

14. Çakalli, H: On Statistical Convergence in topological groups. Pure Appl Math Sci. 43, $27-31$ (1996)

15. Karakus, S: Statistical convergence on probabilistic normed spaces. Math Commun. 12, 11-23 (2007)

16. Karakus, S, Demirci, K, Duman, O: Statistical convergence on intuitionistic fuzzy normed spaces. Chaos Solitons \& Fractals. 35, 763-69 (2008). doi:10.1016/j.chaos.2006.05.046

17. Mursaleen, M: Elements of Metric Spaces. Anamaya Publ., New Delhi (2005)

doi:10.1186/1029-242X-2012-39

Cite this article as: Alotaibi and Alroqi: Statistical convergence in a paranormed space. Journal of Inequalities and Applications 2012 2012:39.

\section{Submit your manuscript to a SpringerOpen ${ }^{\odot}$ journal and benefit from:}

- Convenient online submission

- Rigorous peer review

- Immediate publication on acceptance

- Open access: articles freely available online

- High visibility within the field

- Retaining the copyright to your article

Submit your next manuscript at $\gg$ springeropen.com 